\title{
Learning Variability of Image Feature Appearance Using Statistical Methods
}

\author{
Rodrigo Munguía and Antoni Grau \\ Department of Automatic Control, UPC, \\ c/ Pau Gargallo, 5 E-08028 Barcelona, Spain \\ \{rodrigo.munguia, antoni.grau\} @upc.edu \\ http://webesaii.upc.es
}

\begin{abstract}
Motivated by the problems of vision-based mobile robot map building and localization, in this work, we show that using statistical learning methods the performance of the standard descriptor based methodology for matching image features in a wide base line can be improved. First, we propose two kinds of descriptors for image features and two statistical learning methods. Later, we present a study of the performance of descriptors with and without the statistical learning methods. This work does not pretend to present an exhaustive description of the mentioned methods but to give a good idea the effectiveness of using statistical learning methods together with descriptors for matching image features in a wide base line.
\end{abstract}

\section{Introduction}

Possibly the hardest problem in robotic mapping is the correspondence problem, also known as the data association problem [13]. The correspondence problem is the problem of determining if sensor measurements taken at different points in time correspond to the same physical object in the world. For example two instances of the correspondence problem in robot mapping are closing cycles in large cyclic environment and the kidnapped problem. When a mobile platform moves through its environment a single video camera can be used in order to build a map of its surroundings and to determine its position (absolute or relative). Because in computer vision a great amount of information is available, this information can be used for solve or at least to reduce the challenging correspondence problem. In computer vision, sparse image statistics called features are used in order to create a model that is rich enough to represent the environment and sparse yet to be stored efficiently. A descriptor can be viewed like a distinctive representation of the feature and its variations among the time in a compact way respect the original data without lose of its statistical meaning. In this scenario, the correspondence problem is represented for matching image features descriptors in a wide base line. We understand "wide base line" as a big difference in time and camera point of view, between learning and recognition phases. To address the whole problem descriptors can be very helpful because they can provide distinctive signatures of different locations in space. Furthermore, descriptors have to be as much invariant as possible to changes in scale, rotation, illumination or 
projection (point of view) and algorithms must be efficient and robust to a number of environmental variations such as lighting, shades, and occlusions among others.

Some approaches $[3,4]$ have been presented to address the problem of image features representation for tracking, recognition or reconstruction, the affine invariant descriptors. Generally these methods search for extrema in image scale space for obtaining good candidates locations for detection. Lowe's scale invariant feature transform (SIFT) [5] has showed to match reliably across a wide range of scales and orientation changes; it uses a cascade filtering approach in an isotropic Gaussian scale space to identify points of interest, then it samples gradients to create an orientation histogram represented in a 128-element descriptor. In [6] an approximate version of Kernel Principal Component Analysis (KPCA) was used to estimate features descriptors for wide base line matching.

Invariance to some changes like point of view or illumination are difficult to represent in descriptors created from a single frame since there does not exist a single-view statistic that is invariant with respect to viewpoint or lighting conditions. In applications like structure from motion or robot localization a video stream is available making possible to detect and then track the features across the images with small base line (frame to frame); several trackers $[1,2]$ can be used for this. The correspondence problem is easy in a small base line. While a robot move through their environment and detect image features with its camera, the appearance of the features changes due to natural changes in illumination and changes in the point of view. We can take advantage of matching in a small base line for capture the variations in the appearance of each feature along the time to make the feature descriptors more robust to changes like illumination and point of view.

In our previous work [14] we design a image feature descriptor called ICAD based in ICA (independent component analysis) for matching image features with a wide base line from the incoming video at real time with the feature descriptors previously stored in a data base. Now, in this work we propose to use statistical learning methods for capture variability of image feature appearance. In the learning phase we use small base line tracking methods for capturing the appearance of the image feature along the time, then we build their descriptors, assigning a specific label; these labeled descriptors will be the input to the statistical learning method. In the recognition phase we use the statistical methods to identify the labels of the image features from the incoming video, in real time.

In section 2, we explain the general methodology followed by the implementation of the methods. In section 3 we briefly explain the image feature descriptors and the statistical learning methods used in this work. In section 4 we show the experiments and discuss their results, and finally in section 5 the conclusions and future work are presented.

\section{General Methodology}

We consider the feature matching wide baseline problem under the context of map building and localization of mobile robots. The viewing conditions change drastically between the phases of map building (learning) and localization (recognition). Such changes affect both the domain of the image (deformations of the scene and geometric 
distortion due to changes of the viewpoint) as well as its range (changes in illumination). Such changes are due to both intrinsic properties of the scene (shape, reflectance) and to nuisance factors (illumination, viewpoint).

A feature is a statistic of the image that is intended to make easier the matching process; ideally one would want a statistic feature invariant to all kind of changes. Invariant descriptors to changes like scale or rotation can be created from one single view and they are suitable for many applications, nevertheless in the context of mobile robot, changes like viewpoint or illumination can be appreciably significant between learning and recognition stages. Unfortunately there exist no single view statistic that is invariant respect to the point of view or lighting conditions. On the other hand in the context of mobile robots a high frame-rate video is available during both building and localization. So multiple adjacent views of the same scene are available, as for instance in a video from a moving camera and, at least in theory, the point of view could be explicitly accounted for. Additionally, changes in viewpoint cause irradiance changes that are due to the interplay of reflectance and illumination.

In this work we want to find if the performance of a scheme for matching image features in a wide base line based in a single feature descriptor is increased, if we use multiple descriptors from the same feature taken at different time, for training a statistical learning method, and then use this learning method for the matching process.

So in this work we are interested in the results of a general scheme for matching images features in a wide base line, instead of a specific method performance. Next, a general and modular scheme is presented to address the problem, the idea is to attempt different kind of descriptors and statistical methods of learning and classification, the modularity makes possible to interchange different methods and descriptors. Therefore, the scheme is divided into two stages: learning (map building) and recognition (localization).

\section{Learning phase:}

1. Small base-line tracking: Features are detected and tracked using a conventional small base-line tracker, specifically in this work the Lucas-Kanade Tracker (KLT) was used, but any efficient tracker could be used.

2. Window extraction: For each feature detected a $p$-by- $p$ pixels window around the feature center is extracted, in our work we used a 12-by-12 pixel window. Other window sizes have been tested but the results were worse.

3. Descriptor creation: A descriptor $x_{i}$ is obtained for each window area. A good descriptor has to be as invariant as possible to changes like rotation or scale and insensitive to changes like illumination. We used two statistical techniques: principal component analysis (PCA) and independent component analysis (ICA), but others descriptors like SIFT can be used.

4. Storage in database: For each frame, descriptors have to be scaled and stored in a data base. Descriptors created from the same feature tracked along the time with small base-line are stored with the same label $y_{i}$.

5. Feature class creation: A statistical method is used in order to create and represent the descriptors stored in the database with the same label $y_{i}$ like a unique class $V$ that represent the feature. This feature class $V$ is created with the purpose of capturing the variations in the appearance of the feature along the time. 
The capacity of $V$ to represent these variations depends on the number of descriptors and the changes of scene conditions in which it was created. For this study we used Support Vector Machine (SVM) and variations of K-Nearest Neighbor (KNN).

\section{Recognition phase:}

1. Feature Detection: In order to improve the computational performance in the recognition phase, the same small base-line tracker in the learning phase is used to detect features, but it is not used to track feature candidates to match.

2. Descriptors candidates: For each candidate feature a descriptor $x_{j}$ is created in the same way that it was created in the learning phase.

3. Recognition: The same statistical method used in the learning phase is used now to classify the candidate descriptor $x_{j}$ in the more adequate feature class $V$. Depending on the kind of selected statistical method a quality scheme of correspondence between the candidate descriptor and its associated class can be implemented.

\section{Statistical Methods for Matching Features in a Wide Base-Line}

In this work we employ two image features descriptors: The ICAD [14] and the PCAD, based in [14] too, but using PCA (principal component analysis) instead of ICA (independent component analysis). We used two statistical learning methods: KNN (k-nearest neighbor) and SVM (support vector machine). The idea is comparing the performance of ICAD and PCAD alone, with their performance using them together with KNN and SVM. Consequently in the experiments we are comparing six methods: ICAD, PCAD, SVM-ICA, SVM-PCA, KNN-ICA, and KNN-PCA. In this section first we explain the theoretical bases for ICA, PCA, SVM and KNN, and later, we briefly explain our methods used in this work.

\subsection{PCA and ICA}

Principal Component Analysis (PCA) [7] is a standard statistical tool used to find the orthogonal directions corresponding to the highest variance. It is equivalent to a decorrelation of the data using second-order information. The basic idea in PCA is to find the components $p_{1}, p_{2}, \ldots, p_{\mathrm{n}}$ that explain the maximum amount of variance, by $n$ linearly transformed components. Then, the principal components are given by $p_{i}=w_{i}^{T} X$, where $X=\left[x_{1}, \ldots, x_{\mathrm{m}}\right]^{\mathrm{T}}, x_{i}$ is an observed data vector, and $w_{i}$ is a basis vector (an eigenvector of the sample covariance matrix $E\left\{X X^{\mathrm{T}}\right\}$ ). It can be written, in matrix form, as:

$$
P=W X
$$

where $P=\left[p_{1}, \ldots, p_{n}\right]^{T}$ and $p_{i}$ is a principal component vector.

The Independent Component Analysis (ICA) [8] attempts to go one step further than PCA, by finding the orthogonal matrix $H$ which transforms the data $P$ into $Z$ having $Z_{1}, Z_{2}, \ldots Z_{\mathrm{m}}$ statistically independent. The ICA is thus more general than PCA in 
trying not only to decorrelate the data, but also to find decomposition, transforming the input into independent components. The simplest ICA model, the noise-free linear ICA model, seems to be sufficient for most applications. This model is as follows: ICA of observed random data $X$ consists of estimating the generative model:

$$
X=A S
$$

where $X=\left[x_{1}, \ldots, x_{\mathrm{n}}\right]^{\mathrm{T}} x_{\mathrm{i}}$ is an observed random vector, $S=\left[s_{1}, \ldots, s_{\mathrm{n}}\right]^{\mathrm{T}}, s_{\mathrm{i}}$ is a latent component, and $A$ is the constant mixing matrix. The transform we seek is $B=V W$, then

$$
Z=B X=B A S=C S
$$

If an orthogonal matrix $D$ that transforms the mixed signals $X$ into $Z$ with independent components could be found, and assuming that at least one independent source $s_{k}$ is normally distributed, then $Z=C S$ with $C$ being a non-mixing matrix. The ICA algorithms attempt to find the matrix $B$ which ensures that $Z$ is independent.

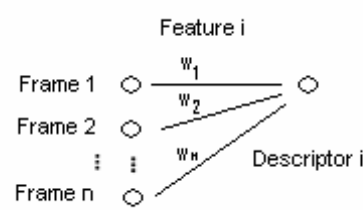

a)

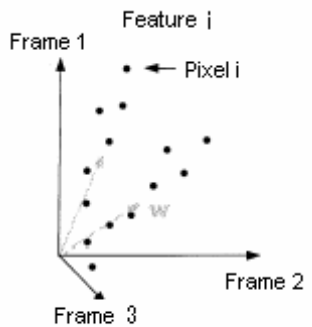

b)

Fig. 1. a) Applying ICA or PCA a matrix is formed where each row is a feature $i$ tracked in frame $n$. b) ICA or PCA finds a weight vector $w$ in the directions of statistical dependencies among the pixel locations. In the case of ICA, data are decorrelated and decompositions found, transforming the input into independent components.

\subsection{ICA and PCA Applied to Window Based Images Features}

If we consider a feature as a window of $p x p$ pixels in a frame, we can organize each feature as a long vector with as many dimensions as number of pixels in the feature. ICA or PCA can be applied to this data organizing each vector into a matrix $X$ where each row is the same image feature for different frames (Fig.1 left). In this approach, images features are random variables and pixels are samples (Fig.1 right).

\subsection{SVM and KNN}

Support Vector Machine (SVM) is a technique used in data classification. The goal of SVM is to produce a model which predicts target value of data instances in the testing set which are given only the attributes.

Given a training set of instance-label pairs $\left(x_{i}, y_{i}\right), i=1, \ldots, l$ where $x_{i} \in R^{n}$ and $y \in\{1,-1\}^{1}$, the SVM [9] require the solution of the following optimization problem: 


$$
\min _{w, b, \xi}\left(\frac{1}{2} w^{t} w+C \sum_{i=1}^{l} \xi_{i}\right) \text {, with } C>0
$$

subject to $y_{i}\left(w^{t} \varphi\left(x_{i}\right)+b\right) \geq 1-\xi_{i}$, where $\xi_{i} \geq 0$.

Here training vectors, $x_{i}$, are mapped into a higher (maybe infinite) dimensional space by the function $\varphi$. Then SVM finds a linear separating hyper-plane with the maximal margin in this higher dimensional space. $C$ is the penalty parameter of the error term. Furthermore, $K\left(x_{i}, x_{j}\right)=\varphi\left(x_{i}\right)^{T} \varphi\left(x_{j}\right)$ is called the kernel function. In this work a radial basis function (RBF) was used like kernel function:

$$
K\left(x_{i} x_{j}\right) \equiv \exp \left(-\gamma\left\|x_{i}-x_{j}\right\|^{2}\right), \gamma>0
$$

The K-nearest neighbor (KNN) is a statistical method of classification well known and very simple, nevertheless has come to demonstrate to be very effective in a wide variety of applications. It works based on minimum distance from the query instance $x_{j}$ to the training samples $\left(x_{i}, y_{i}\right)$, to determine the K-nearest neighbors. After we gather $\mathrm{K}$ nearest neighbors, we take simple majority of these K-nearest neighbors to be the prediction of the query instance.

In this work we use a 5-nearest neighbor; we use a parameter $\alpha$ like a threshold for considering a good match between an $x_{j}$ candidate descriptor and the selected more voted feature class $V$ :

$$
\alpha=\frac{K \sum_{j=1}^{l} d\left(x_{j}, x\right)}{l^{2}}
$$

where $x_{j} \in V, d\left(x_{j}, x\right)$ is the Euclidean distance and $l$ is the number of votes for the more voted class $V$. In this way the average Euclidean distance is used like threshold but is penalized according to the number of votes received for $V$ respect to $K$.

\subsection{ICAD and PCAD Methods}

Many ICA and PCA algorithms are available. A computationally efficient ICA algorithm, called the FastICA [10] algorithm and the PCA Snapshot Method [11] have been chosen for this work.

When the KLT (small base-line tracker) locates a feature (feature $i$ at frame $f$ ), a $p$ by- $p$ pixels window around the feature center is stored as a vector $u_{f i}$ of length $p$-by- $p$, with a distinctive label; in the following frames the feature is tracked and repeating the above process, storing the window with the same label. Immediately vectors with the same label (same feature $i$ ) are regrouped in a matrix $U_{i}=\left[u_{1 \mathrm{i}}, \ldots, u_{\mathrm{ni}}\right]^{\mathrm{T}}$ where $n$ is the number of frames where the feature has been tracked.

Then for each matrix $U$ the ICA or PCA is applied as it has been shown in the section 3.2 along with dimensional reduction selecting the largest eigenvalue to be retained. At the output of the ICA or PCA we obtain a descriptor $q_{i}$ with a dimension 
that equals to the feature window size. The descriptors are stored in a database with a unique label for each feature.

In the recognition phase features are detected but not tracked by the KLT for each incoming frame. Then for each feature detected a window is obtained in the same way than the learning stage and sorted in a vector $v_{i}$, the ICA or PCA is directly applied to this vector without dimensional reduction producing a descriptor $x_{j}$. A fast k-nearest neighbor algorithm is applied to the database in order to look for the 2-nearest neighbor descriptors $q_{i 1}$ and $q_{i 2}$. Be $k_{1}=d\left(x_{j}, q_{i 1}\right)$ and $k_{2}=\mathrm{d}\left(x_{j}, q_{i 2}\right)$ ( $d$ is the Euclidean distance), $k_{1} \leq k_{2}$, and $\eta=k_{1} / k_{2}$, this factor will be used by our algorithm as a threshold for considering a good match between the candidate descriptor $r_{i}$ and its corresponding nearest descriptors $q_{i 1}$ and $q_{i 2}$ in the database. When $\eta$ tends to 0 means a great distance between candidates and, empirically, the results are better.

\subsection{SVM-ICA and SVM-PCA Methods}

We used the LIBSVM [12] for the implementation of the SVM. The method follows exactly the same steps than the feature-class method (section 2): In step 3 (descriptor creation) unlike the ICAD and PCAD methods, the ICA or PCA is applied directly to the vector $u_{f i}$ obtained from de $p$-by- $p$ pixels window (step 2) and stored in the database (step 4). In the output of the ICA or PCA we obtain a descriptor with the same dimension than the pixel window.

For step 5 (feature-class creation) we employ the descriptors-database for training a SVM classifier with a radial basis function (RBF) as a kernel function, equation 4 . The parameters $C=8$ and $\gamma=1$ used in RBF were selected by cross-validation and grid search. For the recognition phase the SVM output model is used to predict the feature class $V$ of the candidate descriptor $x_{j}$, as it has been explained in section 2 (recognition phase).

\subsection{KNN-ICA and KNN-PCA Methods}

For the implementation of KNN we employ a computationally efficient algorithm called approximate nearest neighbor (ANN). The method follows the same steps than SVM-ICA and SVM-PCA except that a training model is not generated from the descriptor-database. Prior to the recognition phase the whole database is loaded in memory by the ANN algorithm. In experiments we used 5-NN. For recognition phase ANN is applied as it has been explained in section 3.3. The threshold $\alpha$ is used to consider a good match between a candidate descriptor $x_{j}$ and the more voted selected feature class $V$ (equation 5).

\section{Experiments}

We have implemented a $\mathrm{C}++$ version of the methods that runs on a PC $2 \mathrm{GHz}$ Pentium IV processor, 512MB RAM. A non-expensive USB Webcam with a maximum resolution of 640-by-480 pixels and $30 \mathrm{fps}$ has been used.

We performed a variety of experiments in order to show the performance of the aforementioned methods. For each method in the learning phase, a video sequence of 
a rigid environment desktop scene was recorded moving the camera slowly and continuously in order to obtain a change of some degrees in the 3D point of view and rotation of the camera. Later, twenty descriptors were created from this video sequence as it was described in section 3. In Fig. 2 (center image) the scene used in the learning phase is showed.

Together with these 20 descriptors, the database contains another 1000 descriptors corresponding to other video sequences. The objective of these experiments consists of observing the response of the methods when a set of descriptors coming from an online video sequence (close to the learning sequence, as it is explained below) will be matched with the descriptors database. The response was observed in four different situations:

- case a) change in $3 \mathrm{D}$ viewpoint with respect to the position in the learning phase and little change in illumination,

- case b) change in 3D point of view plus change in rotation and little change in illumination,

- case c) change in 3D point of view plus change in scale (camera zoom) and little change in illumination,

- case d) change in 3D point of view plus great change in illumination.

Fig. 2(left and right) show some frames used in the recognition phase.
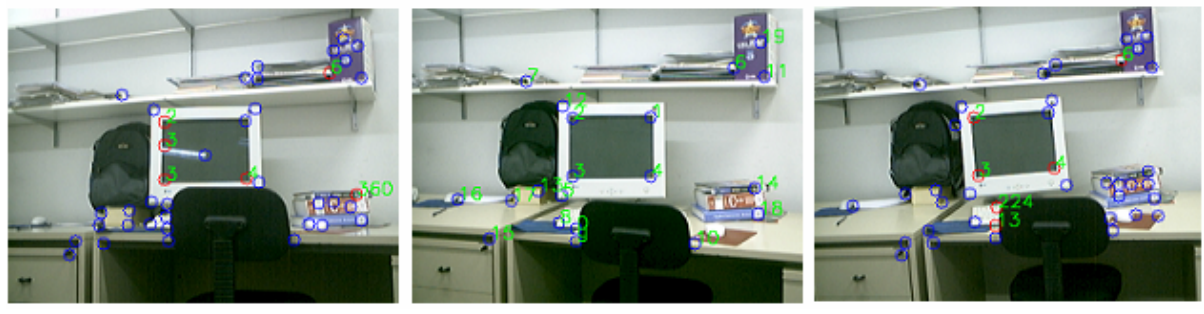

Fig. 2. Descriptors created in the learning phase (central image). Examples of descriptors matched in the recognition phase: (left and right image) we can observe changes in the point of view and illumination.

We define the response of the methods in terms of two measurements:

1) Error of classification: It is the ratio between the percentage of false positive and percentage of classification. We define percentage of false positive as the ratio between the number of features wrong classified and the total of features classified in the scene (correctly or incorrectly). We define percentage of classification as the ratio between the total of features classified in the scene (correctly or incorrectly) and the total that potentially could be matched (we consider only a finite number of possible locations in each frame to be matched, step 1 of recognition phase). For example in the methods based in KNN the percentage of false positive for a threshold $\alpha=0.6$ could be 16 percent but the percentage of classification is 50 percent, consequently we define the error of classification as 0.32. On the other hand in SVM is difficult to establish a threshold for classification, because SVM is a method of the kind "choose the best candidate". Therefore we consider the percentage of classification for SVM 
as 100 percent. For example a 38 percent of false positive in SVM means 38 percent of classification error.

2) Computational cost: We have calculated the time for each frame (or the frequency, it is the same) that the different methods take to classify 30 possible features with the 1000 descriptors that are in the database.

The results for the experiments and the measurements are shown in Table 1.

Table 1. Error of classification for each condition case (a, b, c and d) in the recognition phase and their computational cost. CPU* does not include the time to detect features by KLT tracker.

\begin{tabular}{|c|c|c|c|c|c|c|}
\hline Case & PCAD & ICAD & $\begin{array}{c}\text { SVM- } \\
\text { PCA }\end{array}$ & $\begin{array}{c}\text { SVM- } \\
\text { ICA }\end{array}$ & $\begin{array}{c}\text { KNN- } \\
\text { PCA }\end{array}$ & $\begin{array}{c}\text { KNN- } \\
\text { ICA }\end{array}$ \\
\hline $\mathbf{a}$ & .40 & .35 & .33 & .21 & .26 & .21 \\
\hline $\mathbf{b}$ & .47 & .45 & .44 & .38 & .31 & .32 \\
\hline $\mathbf{c}$ & .47 & .33 & .41 & .28 & .50 & .35 \\
\hline $\mathbf{d}$ & .48 & .47 & .43 & .42 & .37 & .32 \\
\hline CPU & $5.34 \mathrm{~Hz}$ & $5.05 \mathrm{~Hz}$ & $2.32 \mathrm{~Hz}$ & $2.20 \mathrm{~Hz}$ & $5.34 \mathrm{~Hz}$ & $3.84 \mathrm{~Hz}$ \\
\hline CPU* $^{16.30 \mathrm{~Hz}}$ & $10.70 \mathrm{~Hz}$ & $3.22 \mathrm{~Hz}$ & $2.94 \mathrm{~Hz}$ & $21.72 \mathrm{~Hz}$ & $7.09 \mathrm{~Hz}$ \\
\hline
\end{tabular}

\section{Conclusions}

In this work we propose the use of statistical methods for learning variability of image feature appearance, for matching image feature in a wide base line.

In the results of the experiments (Table 1), we can observe a lower error of classification in the methods based in statistical learning (SVM-PCA, SVM-ICA, KNNPCA and KNN-ICA) comparing with ICAD and PCAD methods. Therefore the performance of the ICAD and PCAD methods was increased using statistical learning approaches.

On the other hand, as we expected, ICA-based descriptors show lower error of classification than PCA-based descriptors but computationally the cost for ICA is greater than PCA. We observe a similar computational cost in the case PCAD and ICAD with KNNPCA and KNN-ICA. Finally we also observe that KNN shows better performance than SVM in error of classification as well as in computational cost.

A feature of our approach is the modularity, so in a future work we pretend to use a more robust descriptor like SIFT. Looking the results of this work, we can expect that using SIFT together with a scheme of statistical learning we can improve the performance of SIFT alone, for applications like a mobile robot where an incoming stream video is available.

\section{References}

1. Shi, J., Tomasi, C.: Good features to track, Proc. IEEE CVPR, 1994.

2. Harris, C., Stephens, M.: A combined corner and edge detector, Alvey Vision Conf., 1988.

3. Mikolajczyk, K., Schmid, C.: An affine invariant interest point detector, Proc. ECCV, 2002. 
4. Lowe, D.: Object recognition from local scale-invariant features, Proc. ICCV, Corfu, Greece, September 1999.

5. Lowe, D.: Distinctive image features from scale-invariant keypoints, International Journal of Computer Vision, 60 (2): 91-110, 2004.

6. Meltzer, J., Yang, M H., Gupta, R., Soatto, S.: Multiple view feature descriptors from image sequences via kernel principal component analysis, Proc. ECCV, 2004.

7. Jolliffe, I.T.: Principal Component Analysis. Springer Verlag, 1986.

8. Comon, P.: Independent component analysis, a new concept?, Signal Processing, Elsevier, 36(3): 287-314, April 1994.

9. Boser, B. E., Guyon, I., Vapnik, V.: A training algorithm for optimal margin classifiers, Proc. of the Fifth Annual Workshop on Computational Learning Theory 5, pp. 144-152, 1992.

10. Hyvarinen, A., Oja, E.: A fast fixed-point algorithm for independent component analysis, Neural Computation, 9 (7): 1483-1492, 1997.

11. Sirovich, L.: Turbulence and the dynamics of coherent structures, Part 1: Coherent Structure, Quarterly of Applied Mathematics, 45 (3): 561-571, October 1987.

12. Chih-Chung Chang, Chih-Jen Lin: LIBSVM : a library for support vector machines, 2001. Software available at http://www.csie.ntu.edu.tw/ cjlin/libsvm.

13. Sebastian Thrun: Robotic Mapping CMU-CS-02-111.2000.

14. R. Munguía, A. Grau, A. Sanfeliu: Matching Images Features in a Wide Base Line with ICA Descriptors. IAPR 18th International Conference for Pattern Recognition, ICPR 2006, Honk Kong, to be appeared. 